Bundesgesundheitsbl 2020 · 63:1168-1176 https://doi.org/10.1007/s00103-020-03207-7 Online publiziert: 21 . August 2020

(C) Der/die Autor(en) 2020

\author{
Anja Hartmann' · Jana Rückmann ${ }^{2} \cdot$ Antje Tannen $^{2}$ \\ ${ }^{1}$ Koordinierungsstelle Nationaler Aktionsplan Gesundheitskompetenz, Hertie School Berlin's University of \\ Governance, Berlin, Deutschland \\ ${ }^{2}$ Institut für Gesundheits- und Pflegewissenschaft, Charité Universitätsmedizin Berlin, Berlin, Deutschland
}

\title{
Individuelle Gesundheitskompetenz von Lehrkräften und deren (Un)Sicherheit im Umgang mit chronisch erkrankten Schulkindern und Notfallsituationen
}

\section{Hintergrund}

Gesundheitskompetenz gilt als eine Schlüsseldeterminante im Hinblick auf die Gesundheitssicherung [1] und wird als zentrales Element in der Gesundheitsförderung diskutiert [2]. Zudem wurde in den letzten Dekaden intensiv an der Konzeptentwicklung und Operationalisierung von Gesundheitskompetenz gearbeitet [3-8]. Schließlich wurde im Jahr 2010 das European Health Literacy Consortium (EU-HLC) mit dem Ziel gegründet, Daten zur Gesundheitskompetenz bei den EUBürger/-innen zu erheben. Das zugrunde liegende konzeptuelle Modell basiert auf allgemeiner Literalität (Lese- und Schreibfähigkeit) und integriert Wissen, Motivation und Kompetenzen, um gesundheitsrelevante Informationen in den Domänen Krankheitsbewältigung, Prävention und Gesundheitsförderung $\mathrm{zu}$ finden, zu verstehen, zu beurteilen und umzusetzen [8].

Erstellung der Arbeit im Rahmen der Bachelorthesis an der Charité Universitätsmedizin, Institut für Gesundheits- und Pflegewissenschaft.
Mit dem auf diesem Modell aufbauenden Messinstrument „European Health Literacy Survey Questionnaire“ (HLSEU-Q) wurde im Jahr 2011 erstmals die Gesundheitskompetenz der Bevölkerung von acht europäischen Mitgliedstaaten des EU-HLC erhoben (HLS-EU 2012). Im Ergebnis weist nahezu jede/-r zweite befragte Europäer/-in eine eingeschränkte Gesundheitskompetenz (GK) auf. AuBerdem wurden vulnerable Gruppen mit einem eingeschränkten GK-Niveau identifiziert, darunter finanziell, bildungsbezogen oder durch chronische Erkrankungen benachteiligte Menschen. Die Ergebnisse der nationalen Studien verhalten sich ähnlich zu dem Verteilungsmuster des EU-Durchschnitts bzw. des deutschen HLS-EU Ergebnisses [9].

Nicht zuletzt aufgrund dieser Befunde wurde im Jahr 2018 der „Nationale Aktionsplan Gesundheitskompetenz" (NAP) als Ergebnis der „Allianz für Gesundheitskompetenz" in Berlin vorgestellt [10]. Es folgten bislang 5 Strategiepapiere (Stand: August 2019), das erste mit der Forderung, „... das Erziehungs- und Bildungssystem in die Lage [zu] versetzen, die Förderung von Gesundheitskompetenz so früh wie möglich im Lebenslauf beginnen [zu lassen]“ [11]. Denn gera- de den frühen Lebensphasen wird ein hohes Potenzial für die Entwicklung gesundheitsbezogener Ressourcen und Resilienz sowie Gesundheitskompetenz zugesprochen [12, 13]. Zudem werden im ersten Strategiepapier konkrete Handlungsempfehlungen zur Umsetzung der Forderung formuliert. Allerdings wurde die individuelle Gesundheitskompetenz der Lehrkräfte bzw. weiterer Akteure im Bildungssystem nicht berücksichtigt. Mit Blick auf die einschlägige Literatur gilt diese jedoch als zentraler Faktor für ein professionelles Handeln im Rahmen der Förderung von Gesundheitskompetenz bei den Schüler/-innen [12, 14-16].

Die Notwendigkeit einer ausreichenden Gesundheitskompetenz der Lehrkräfte wird zudem durch die sog. neue Morbidität [17] unterstrichen, welche nicht durch neue Erkrankungen, sondern eher durch eine größere Relevanz bestimmter Erkrankungen gekennzeichnet ist. Ähnlich wie bei der erwachsenen Bevölkerung steigen auch bei den Heranwachsenden die Prävalenzen von chronischen Erkrankungen wie Neurodermitis, Asthma bronchiale, Diabetes mellitus sowie von psychischen Problemen bzw. Auffälligkeiten [18-22]. 
Tab. 1 Stichprobenbeschreibung: Lehrkräfte an allgemeinbildenden Schulen in Brandenburg und Hessen

\begin{tabular}{l|l}
\hline & Häufigkeiten in \% (N) und Verteilungen \\
\hline $\begin{array}{l}\text { Stichprobe (N) } \\
\text { Bildungsstufe }\end{array}$ & 420 \\
$\begin{array}{l}\text { Grundstufe } \\
\text { Sekundarstufe 1 }\end{array}$ & $17,6 \%(74)$ \\
\hline Sekundarstufe 2 & $30,7 \%(129)$ \\
Grund- und Sekundarstufe 1 & $3,1 \%(13)$ \\
\hline Sekundarstufe 1 und 2 & $13,3 \%(56)$ \\
\hline Ohne Angabe & $29,8 \%(125)$ \\
\hline Geschlecht & $5,5 \%(23)$ \\
\hline Weiblich & \\
\hline Männlich & $75,5 \%(317)$ \\
\hline Ohne Angabe & $22,4 \%(94)$ \\
\hline Alter (in Jahren) & $2,1 \%(9)$ \\
\hline Minimum & \\
\hline Maximum & 26 \\
\hline MW \pm SD & 65 \\
\hline Ohne Angabe & $46,02 \pm 10,844$ \\
\hline MW Mittelwert, SD Standardabweichung & $3,6 \%(15)$ \\
\hline
\end{tabular}

Insbesondere der Anstieg von psychischen Auffälligkeiten - sowohl bei den Schüler/innen als auch bei deren Eltern - und damit verbundenen familiären $\mathrm{Be}$ lastungen verlangt von den Lehrkräften, dass sie diese früh erkennen, adäquate Unterstützung anbieten und eine Vermittlerposition zwischen Schule und Hilfesystem wahrnehmen [23].

Die gesundheitspolitischen Handlungsempfehlungen des Strategiepapiers fokussieren vor allem das Setting Schule als wichtigen Sozialisationsort der jungen Menschen. Neben der guten Erreichbarkeit der schulpflichtigen Kinder und Jugendlichen steht das Setting Schule auch für einen Ort des Lernens und des Kompetenzerwerbs. An diesem Ort besteht für Lehrkräfte am ehesten die Chance, auf die Entwicklung gesundheitsförderlicher Verhaltensweisen der jungen Menschen einwirken zu können. Dabei hat die individuelle Gesundheitskompetenz der Lehrkräfte zum einen Auswirkungen auf deren Fähigkeit, in der direkten Vermittlung von Gesundheitsbildung zu agieren, zum anderen werden auch indirekt gesundheitsrelevante Botschaften an die Schüler/-innen gesendet. Die Gesundheitskompetenz der Lehrkräfte ist ausschlaggebend für den Er- folg schulischer Gesundheitsförderung und beeinflusst die Gesundheitskompetenz der Heranwachsenden [12, 14, 15]. Hinzu kommt: Durch eine Vielzahl an Inklusionsbestrebungen [24] sowie die Umstellung auf den Ganztagsbetrieb an allgemeinbildenden Schulen haben Lehrkräfte eine erhöhte Kontakthäufigkeit mit chronisch erkrankten Kindern und Jugendlichen, die eine Erweiterung ihres Aufgabenspektrums erfordern, so zum Beispiel die gesundheitliche Versorgung im Bedarfsfall [25, 26]. Zu der verstärkten Konfrontation mit Gesundheitsfragen im Schulalltag stellt eine erhöhte soziale und kulturelle Heterogenität der Schülerschaft ebenfalls hohe Anforderungen an Lehrkräfte, bspw. in Form veränderter Leistungsansprüche oder psychischer Belastungen. Diese Mehrfachbelastung kann langfristig zu einer gesundheitlichen Beeinträchtigung der Lehrkräfte selbst führen [27].

In der Folge gelten beschwerdefreie Lehrkräfte inzwischen eher als Minderheit; mindestens $20 \%$ der an allgemeinbildenden Schulen unterrichtenden Lehrkräfte weisen gravierende Einschränkungen ihrer Gesundheit auf; psychosomatische Beschwerden kommen dabei überhäufig vor [28]. Im
Vergleich zu anderen Berufsgruppen liegt bei dieser Personengruppe eine erhöhte Befundhäufigkeit von psychischen Erkrankungen, zum Beispiel depressiven Störungen, vor. Außerdem gelten affektive Störungen wie Erschöpfungssyndrome oder Depression als Hauptursache für die jährlich steigende Frühpensionierungsrate in dieser Berufsgruppe [29]. Eine hohe Gesundheitskompetenz der Lehrkräfte wäre demnach nicht nur für die Gesundheit der Schüler/-innen, sondern auch für den Erhalt und die Förderung der Lehrergesundheit von hoher Relevanz.

Neben den genannten Kompetenzen im Umgang mit Gesundheitsinformationen werden Lehrkräfte an allgemeinbildenden Schulen auch mit konkreten akut auftretenden Krankheitssymptomen und Notfällen sowohl bei gesunden als auch insbesondere im Zuge der Inklusion bei chronisch kranken Schüler/-innen konfrontiert. Oftmals sind sie ungenügend darauf vorbereit, die Situation fachkundig einzuschätzen, adäquat zu reagieren oder eine Entscheidung über die Weiterbehandlung zu treffen, da das nicht $\mathrm{zu}$ ihren Kernaufgaben zählt [30, 31]. Genaue Erkenntnisse darüber, wie sicher sich die Lehrkräfte im Umgang mit gesundheitlichen Notfällen fühlen und wo Unterstützungsbedarf besteht, fehlen jedoch in Deutschland.

Diese offenen Fragen aufgreifend, soll in der vorliegenden Untersuchung ermittelt werden, wie hoch das Niveau der Gesundheitskompetenz der Lehrkräfte ist und ob es einen Zusammenhang zwischen der individuellen Gesundheitskompetenz von Lehrkräften sowie deren Sicherheit im Umgang mit chronisch erkrankten Schüler/-innen und Notfallsituationen gibt.

\section{Forschungsfokus und Methoden}

Die folgenden Befunde stammen aus der wissenschaftlichen Evaluation der Einführung von Schulgesundheitspflegenden an allgemeinbildenden Schulen (SPLASH; [32, 33]). Im Rahmen dieser Begleitforschung erfolgte im Jahr 2017 an den $N=30$ am Projekt beteiligten Modellschulen in Brandenburg und Hessen eine standardisierte schriftliche Lehr- 
Bundesgesundheitsbl 2020 • 63:1168-1176 https://doi.org/10.1007/s00103-020-03207-7

(c) Der/die Autor(en) 2020

\section{A. Hartmann · J. Rückmann · A. Tannen}

\section{Individuelle Gesundheitskompetenz von Lehrkräften und deren (Un)Sicherheit im Umgang mit chronisch erkrankten Schulkindern und Notfallsituationen}

\section{Zusammenfassung}

Hintergrund. Gesundheitskompetenz (GK) gilt als eine Schlüsseldeterminante im Hinblick auf die Gesundheitssicherung. Die Förderung von GK sollte so früh wie möglich beginnen, um späteren Gesundheitsrisiken vorzubeugen. Im Setting Schule haben Lehrkräfte hierbei eine zentrale Rolle, denn sie werden durch den Ganztagsschulbetrieb sowie die Inklusionsbestrebungen immer häufiger mit Gesundheitsproblemen Heranwachsender konfrontiert. Gleichwohl kann festgestellt werden, dass eine Vielzahl der Lehrkräfte selbst erhebliche Gesundheitsprobleme aufweist, u. a. bedingt durch hohe Arbeitsbelastungen. Fragestellung. In dieser Arbeit wird das Niveau der GK von Lehrkräften ermittelt und ein möglicher Zusammenhang zwischen individueller GK der Lehrkräfte und deren Sicherheit im Umgang mit chronisch erkrankten Schulkindern untersucht. Methoden. Es erfolgte eine Sekundärdatenanalyse von Daten aus einer quantitativen Befragung von $n=420$ Lehrkräften. Zur Selbsteinschätzung ihrer GK wurde der „European Health Literacy Survey Questionnaire" (HLS-EU-Q16) verwendet. Weiterhin erfolgten Korrelationsanalysen zwischen der GK und dem Grad der Unsicherheit im Umgang mit ausgewählten Gesundheitsproblemen. Ergebnisse. Mehr als die Hälfte der Befragten weist eine eingeschränkte GK auf. Gleichzeitig ist ein deutlicher Zusammenhang zwischen GK und der Sicherheit im Umgang mit chronisch erkrankten Schüler/-innen festzustellen.
Insbesondere im Umgang mit psychischer Gesundheit bestehen Unsicherheiten.

Diskussion. Es besteht eine Diskrepanz zwischen den Anforderungen an die Lehrkräfte im Hinblick auf die Bewältigung von Gesundheitsproblemen im Setting Schule und ihrer dafür benötigten Gesundheitskompetenz und Souveränität im Umgang mit chronischen und psychischen Beeinträchtigungen. Die genannten Aspekte können einen Einfluss auf die kindliche GK sowie die gesundheitliche Entwicklung der Heranwachsenden nehmen.

Schlüsselwörter

Gesundheitsförderung · Schulische Gesundheit · Settingansatz · Nationaler Aktionsplan . Psychische Gesundheit

\section{Individual teacher's health literacy and their safety in dealing with chronically ill pupils and emergencies}

\section{Abstract}

Background. Health literacy $(\mathrm{HL})$ is regarded as a key determinant in health promotion. The support of HL should begin as early as possible to prevent later health problems. In the school setting, teachers play an important role. Due to all-day school and inclusion efforts, teachers are increasingly faced with the health problems of pupils. At the same time, many teachers show their own significant health problems, often due to an enormous workload.

Objectives. This study assesses the level of the individual teacher's $\mathrm{HL}$ and examines possible relationships between the individual
$\mathrm{HL}$ of teachers and their level of uncertainty in dealing with chronically ill pupils.

Methods. A secondary data analysis of a quantitative survey of $n=420$ teachers was conducted. A self-assessment of the teacher's HL level was done using HLS-EU-Q16. Furthermore, correlation analyses between the $\mathrm{HL}$ and the level of insecurity of teachers in dealing with selected health problems were made.

Results. More than half of the teachers showed a limited level of $\mathrm{HL}$ and an association between low level of $\mathrm{HL}$ and uncertainty in dealing with chronically ill pupils were found.
In particular, mental health issues caused difficulties among the teachers.

Discussion. There is a large gap between complex health-related demands on the teachers and their required health literacy as well as sovereignty in dealing with chronic and mental impairments in the school setting. These deficits will influence the children's HL and the health development of adolescents.

Keywords

Health promotion $\cdot$ School health $\cdot$ Setting approach · National Action Plan · Mental health kräftebefragung $(N=1279)$. Die Teilnahme an der Befragung war freiwillig, anonym und nach vorheriger schriftlicher Aufklärung. Die Studie wurde durch die zuständigen Landesministerien genehmigt und es lag ein ethisches Clearing durch die Ethikkommission der Deutschen Gesellschaft für Pflegewissenschaft vor (Antrag Nr. 16-025). Insgesamt konnten $N=420$ Fragebögen in die Datenanalyse eingeschlossen werden, das entspricht einer Rücklaufquote von 32,8 \% [33]. Im Fokus der Befragung stand die Erfassung der Gesundheits- kompetenz der Lehrkräfte mittels des HLS-EU-Q16, einer Kurzversion des HLS-EU-Q47 [34]. Der HLS-EU-Q47 basiert auf dem konzeptuellen Modell von Sørensen et al. [8]. Die deutsche Version erreicht eine akzeptable Reliabilität (Cronbachs Alpha zwischen 0,78 und 0,93). Die Kurzversion, die in dieser Studie verwendet wurde, weist zudem eine ausreichende Inhaltsvalidität auf [9]. Aus den Antworten der 16 Items wird ein individueller Summenscore gebildet, welcher zwischen 0 (geringste Gesundheitskompetenz) und 16 (ma- ximale Gesundheitskompetenz) liegen kann. Aus diesem Summenscore wurden 3 Kategorien gebildet: ausreichend (13-16 Punkte), problematisch [9-12] und inadäquat (0-8 Punkte; [35]).

Des Weiteren wurden die Lehrkräfte befragt, inwiefern Schwierigkeiten im Umgang mit bestimmten Erkrankungen sowie mit Notfallsituationen bei Schüler/-innen mit chronischen Erkrankungen bestehen. Dazu konnten die Lehrkräfte zu häufigen pädiatrischen Erkrankungen sowie potenziellen Notfallsituationen auf einer 4-Punkt- 
Tab. 2 Gesundheitskompetenzlevel der Lehrkräfte (SPLASH-Studie 2017 [32]) im Vergleich zur Referenzpopulation (GEDA-Studie 2014 [37], hohes Bildungsniveau)

\begin{tabular}{|c|c|c|c|c|c|}
\hline & Level 1: ausreichend (\%) & Level 2: problematisch (\%) & Level 3: inadäquat (\%) & $p$-Wert ${ }^{\mathrm{a}}$ & Gesamt $N$ \\
\hline Frauen & 50,7 & 38,4 & 10,9 & n.s. & 284 \\
\hline Männer & 49,4 & 41,4 & 9,2 & - & 87 \\
\hline Alter 26-35 & 50,6 & 39,3 & 10,1 & n.s. & 89 \\
\hline Alter $36-45$ & 55,1 & 35,9 & 9,0 & - & 79 \\
\hline Alter $46-55$ & 42,9 & 41,1 & 16,1 & - & 112 \\
\hline Alter 56-65 & 55,2 & 39,1 & 5,1 & - & 87 \\
\hline Grundstufe & 48,4 & 39,1 & 12,5 & $* *$ & 64 \\
\hline Sekundarstufe 1 & 45,5 & 46,4 & 8,0 & - & 112 \\
\hline Sekundarstufe 2 & 0,0 & 91,7 & 8,3 & - & 12 \\
\hline Grund- und Sekundarstufe 1 & 63,5 & 25,0 & 11,5 & - & 52 \\
\hline Sekundarstufen 1 und 2 & 53,4 & 34,5 & 12,1 & - & 116 \\
\hline Gesamt Lehrkräfte(SPLASH) & 50,1 & 39,3 & 10,6 & - & 377 \\
\hline GEDA (hohes Bildungsniveau) & 59,6 & 31,1 & 9,3 & - & 2094 \\
\hline \multicolumn{6}{|c|}{ 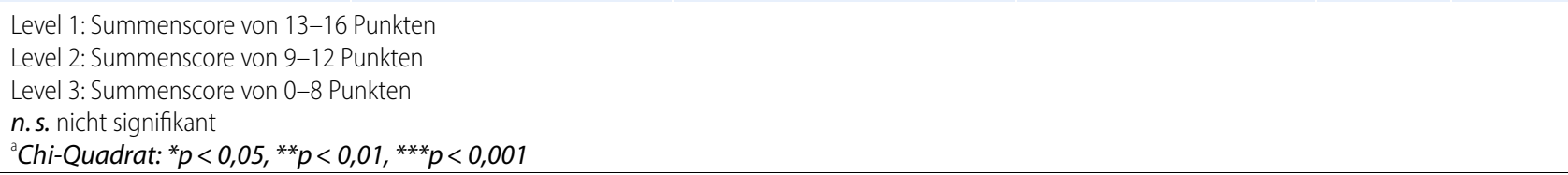 } \\
\hline
\end{tabular}

Likertskala einschätzen, wie sicher sie sich jeweils im Umgang damit fühlen. Für die Korrelationen wurde ein Summenscore gebildet. Dazu wurde die Antwortmöglichkeit „sehr sicher" mit 4 codiert, „eher sicher“ mit 3, „eher unsicher" mit 2 sowie „sehr unsicher" mit 1. Demnach entspricht ein hoher Summenscore einem sicheren Umgang mit chronisch erkrankten Schüler/-innen bzw. für einen sicheren Umgang mit Notfallsituationen. Beide Skalen zur Selbsteinschätzung der Lehrkräfte in Bezug auf den Umgang mit bestimmten Erkrankungen sowie mit medizinischen Notfällen wurden im Rahmen der Studie literaturbasiert entwickelt und in einer interprofessionellen Arbeitsgruppe konsentiert. Neben der univariaten Analyse von Häufigkeitsverteilungen (bei kategorialen und ordinalen Skalenniveaus) und Verteilungsparametern (bei metrischen Skalenniveaus) wurden multiple Regressionsanalysen für den Zusammenhang zwischen der "Gesundheitskompetenz" (Summenscore) und der „Sicherheit im Umgang mit chronisch erkrankten Schüler/-innen“(Summenscore) bzw. der „Sicherheit im Umgang in Notfallsituationen" (Summenscore) durchgeführt. Es wurde jeweils für Alter (metrisch) und Geschlecht (dichotom) adjustiert.
Die Voraussetzungen für die linearen Regressionsanalysen wurden durch die Analyse der Residuen geprüft. Dazu wurden ein Histogramm und der Q-QPlot der Residuen betrachtet. Obwohl beide Diagramme auf Verletzungen der Voraussetzungen für die lineare Regressionsanalyse hindeuten, erscheinen diese nicht so gravierend, dass die Analysen $\mathrm{zu}$ falschen Ergebnissen führen.

Sämtliche Analysen wurden mit dem Statistikprogramm IBM ${ }^{\circledR}$ SPSS $^{\circledR}$ Statistics Version 24 für Mac durchgeführt.

\section{Ergebnisse}

\section{Stichprobenbeschreibung}

Insgesamt konnten, bei einer Rücklaufquote von $32,8 \%, N=420$ Fragebögen in die Datenauswertung eingeschlossen werden. Die meisten Lehrkräfte unterrichteten in der Sekundarstufe I und II (63,6\%). Über 2 Drittel der befragten Personen sind weiblich (75,5\%). Die Befragten waren im Mittel 46 Jahre alt (•Tab. 1).

\section{Gesundheitskompetenz der Lehrkräfte}

Die Befunde zur Ausprägung der Gesundheitskompetenz zeigen: Die Hälfte der befragten Lehrkräfte wies eine ausreichende Gesundheitskompetenz (50,1\%) auf $(39,3 \%$ problematisch; $10,6 \%$ inadäquat; • Tab. 2). Unterschiede in der Gesundheitskompetenz bedingt durch Alter oder Geschlecht waren nicht signifikant. Eindeutige Unterschiede in Abhängigkeit vom Schultyp lassen sich nicht erkennen.

Die größten Schwierigkeiten haben die Befragten in den Domänen „Prävention“, gefolgt von „Krankheitsbewältigung" und "Gesundheitsförderung" (- Tab. 3). Im Rahmen der Domäne „Prävention“ stellt die Aufgabe der Beurteilung der Vertrauenswürdigkeit von Informationen über Gesundheitsrisiken in den Medien die größte Schwierigkeit für die befragten Lehrkräfte dar (51,6\%). In der Domäne Krankheitsbewältigung fällt es den Befragten besonders schwer zu beurteilen, wann sie eine zweite ärztliche Meinung einholen sollten (47,1\%). Innerhalb der Domäne „Gesundheitsförderung " fällt es den Befragten besonders schwer, Informationen zu Verhaltensweisen $\mathrm{zu}$ finden, die ihr psychisches Wohlbefinden stärken $(23,1 \%)$.

Neben den 3 Domänen der Gesundheitskompetenz (Krankheitsbewältigung, Prävention und Gesundheitsförderung) lassen sich in dem Modell von Sørensen et al. [8] auch noch 4 


\begin{tabular}{|c|c|c|c|c|c|c|c|}
\hline Domäne & Item & $\begin{array}{l}\text { Auf einer Skala von sehr einfach bis sehr } \\
\text { schwierig, wie einfach schätzen Sie ein ... }\end{array}$ & $\begin{array}{l}\text { Sehr ein- } \\
\text { fach } \\
\text { (in \%) }\end{array}$ & $\begin{array}{l}\text { Ziemlich } \\
\text { einfach } \\
\text { (in \%) }\end{array}$ & $\begin{array}{l}\text { Ziemlich } \\
\text { schwierig } \\
\text { (in \%) }\end{array}$ & $\begin{array}{l}\text { Sehr } \\
\text { schwierig } \\
\text { (in \%) }\end{array}$ & $N$ \\
\hline \multirow[t]{7}{*}{$\begin{array}{l}\text { Krankheits- } \\
\text { bewältigung }\end{array}$} & Q1 & $\begin{array}{l}\text { Informationen über Therapien für Krankheiten, } \\
\text { die Sie betreffen, zu finden? }\end{array}$ & 12,9 & 63,3 & 22,5 & 1,3 & 387 \\
\hline & Q2 & $\begin{array}{l}\text { Herauszufinden, wo Sie professionelle Hilfe er- } \\
\text { halten, wenn sie krank sind? }\end{array}$ & 16,2 & 57,6 & 24,5 & 1,8 & 396 \\
\hline & Q3 & Zu verstehen, was ihr Arzt Ihnen sagt? & 22,3 & 64,2 & 13,3 & 0,3 & 399 \\
\hline & Q4 & $\begin{array}{l}\text { Die Anweisungen Ihres Arztes oder Apothekers } \\
\text { zur Einnahme der verschriebenen Medikamente } \\
\text { zu verstehen? }\end{array}$ & 48,5 & 50 & 1,3 & 0,3 & 400 \\
\hline & Q5 & $\begin{array}{l}\text { Zu beurteilen, wann Sie eine zweite Meinung } \\
\text { von einem anderen Arzt einholen sollten? }\end{array}$ & 11,9 & 41 & 42,8 & 4,3 & 395 \\
\hline & Q6 & $\begin{array}{l}\text { Mithilfe der Informationen, die Ihnen der Arzt } \\
\text { gibt, Entscheidungen bezüglich Krankheiten zu } \\
\text { treffen? }\end{array}$ & 9,7 & 55,1 & 33,9 & 1,3 & 383 \\
\hline & Q7 & $\begin{array}{l}\text { Den Anweisungen Ihres Arztes oder Apothekers } \\
\text { zu folgen? }\end{array}$ & 35,8 & 58,9 & 5,3 & 0 & 399 \\
\hline \multirow[t]{5}{*}{ Prävention } & Q8 & $\begin{array}{l}\text { Informationen über Unterstützungsmöglichkei- } \\
\text { ten bei psychischen Problemen, wie Stress oder } \\
\text { Depression, zu finden? }\end{array}$ & 11,1 & 40,1 & 40,9 & 7,9 & 379 \\
\hline & Q9 & $\begin{array}{l}\text { Gesundheitswarnungen vor Verhaltensweisen } \\
\text { wie Rauchen, wenig Bewegung oder übermäßi- } \\
\text { ges Trinken zu verstehen? }\end{array}$ & 62,2 & 35,8 & 1,8 & 0,3 & 399 \\
\hline & Q10 & $\begin{array}{l}\text { Zu verstehen, warum Sie eine Vorsorgeuntersu- } \\
\text { chung brauchen? }\end{array}$ & 53,4 & 43,4 & 3,2 & 0 & 401 \\
\hline & Q11 & $\begin{array}{l}\text { Zu beurteilen, ob die Informationen über Ge- } \\
\text { sundheitsrisiken in den Medien vertrauenswür- } \\
\text { dig sind? }\end{array}$ & 9,5 & 38,8 & 47,1 & 4,5 & 399 \\
\hline & Q12 & $\begin{array}{l}\text { Aufgrund von Informationen aus den Medien } \\
\text { zu entscheiden, wie Sie sich vor Krankheiten } \\
\text { schützen können? }\end{array}$ & 11,1 & 48,6 & 37,5 & 2,8 & 395 \\
\hline \multirow[t]{4}{*}{$\begin{array}{l}\text { Gesundheits- } \\
\text { förderung }\end{array}$} & Q13 & $\begin{array}{l}\text { Informationen über Verhaltensweisen zu finden, } \\
\text { die gut für Ihr psychisches Wohlbefinden sind? }\end{array}$ & 18,8 & 58 & 21,6 & 1,5 & 393 \\
\hline & Q14 & $\begin{array}{l}\text { Gesundheitsratschläge von Familienmitgliedern } \\
\text { oder Freunden zu verstehen? }\end{array}$ & 34,8 & 55,3 & 9,1 & 0,8 & 396 \\
\hline & Q15 & $\begin{array}{l}\text { Informationen in den Medien darüber, wie Sie } \\
\text { Ihren Gesundheitszustand verbessern können, } \\
\text { zu verstehen? }\end{array}$ & 24 & 56,7 & 18,2 & 0,5 & 390 \\
\hline & Q16 & $\begin{array}{l}\text { Zu beurteilen, welche Alltagsgewohnheiten mit } \\
\text { Ihrer Gesundheit zusammenhängen? }\end{array}$ & 28,6 & 56,3 & 14,3 & 0,8 & 398 \\
\hline
\end{tabular}

Dimensionen im Zusammenhang mit Gesundheitsinformationen unterscheiden (Informationen finden, verstehen, beurteilen und anwenden). Betrachtet man die Dimensionen der Gesundheitskompetenz der Lehrkräfte, so fällt es den Befragten besonders schwer, Informationen zu beurteilen, gefolgt vom Finden, Anwenden und Verstehen von Informationen (•Tab. 3). In der Dimension „Beurteilen“ fällt es mehr als der Hälfte der Befragten schwer zu beurteilen, ob die Informationen über Gesundheitsrisiken in den Medien vertrauenswürdig sind (51,6\%). In der Dimension „Finden" bestehen die größten Probleme beim Ermitteln von Informationen über Unterstützungsmöglichkeiten bei psychischen Problemen, wie Stress oder Depression (48,8\%). In der Dimension "Anwenden“ fällt es den Befragten besonders schwer, auf der Grundlage von Informationen aus den Medien zu entscheiden, wie sie sich vor Krankheiten schützen können (40,3\%). Die größten Probleme ergeben sich beim „Verstehen“ von Medieninformationen zu Möglichkeiten der Gesundheitsförderung (18,7\%).

Fehlende Angaben des HLS-EU-Q16 variieren zwischen $4,5 \%$ und $9,8 \%$. Die meisten finden sich bei Frage 8 (Informationen über Unterstützungsmöglichkeiten bei psychischen Problemen wie Stress oder Depression finden). 
Tab. 4 Häufigkeitsverteilungen für die Sicherheit im Umgang mit chronisch erkrankten

Schüler/-innen sowie in Notfallsituationen (SPLASH-Studie 2017)

\begin{tabular}{|l|l|l|l|l|}
$\begin{array}{l}\text { Sehr sicher } \\
\text { (in \%) }\end{array}$ & $\begin{array}{l}\text { Eher sicher } \\
\text { (in \%) }\end{array}$ & $\begin{array}{l}\text { Eher unsi- } \\
\text { cher } \\
\text { (in \%) }\end{array}$ & $\begin{array}{l}\text { Sehr unsi- } \\
\text { cher } \\
\text { (in \%) }\end{array}$ & $N$ \\
\hline
\end{tabular}

Sicherheit im Umgang mit chronisch erkrankten Schüler/-innen

\begin{tabular}{|c|c|c|c|c|c|}
\hline Neurodermitis & 8,7 & 41,7 & 35,2 & 14,4 & 403 \\
\hline Asthma bronchiale & 4,2 & 25,4 & 45,2 & 25,2 & 405 \\
\hline Diabetes mellitus & 3,7 & 21,8 & 44,4 & 30 & 403 \\
\hline Essstörungen & 4 & 24,2 & 51,6 & 20,2 & 405 \\
\hline $\begin{array}{l}\text { Schulangst und } \\
\text { Schulphobie }\end{array}$ & 4,9 & 25,1 & 47,9 & 22,1 & 407 \\
\hline Depression & 2,7 & 17 & 50 & 30,3 & 406 \\
\hline ADHS & 7,3 & 48 & 36,6 & 8 & 410 \\
\hline \multicolumn{6}{|c|}{ Sicherheit im Umgang in Notfallsituationen } \\
\hline $\begin{array}{l}\text { Beginnende allergische } \\
\text { Reaktion }\end{array}$ & 2,7 & 25,8 & 48,2 & 23,4 & 415 \\
\hline Allergischer Schock & 1,7 & 9,5 & 38,8 & 50 & 410 \\
\hline $\begin{array}{l}\text { Notfallsituation bei ange- } \\
\text { borenem Herzfehler }\end{array}$ & 1 & 6,3 & 31,4 & 61,3 & 411 \\
\hline Asthmaanfall & 3,6 & 22,7 & 38,1 & 35,7 & 415 \\
\hline $\begin{array}{l}\text { Unterzuckerung bei Dia- } \\
\text { betes }\end{array}$ & 3,9 & 21,4 & 41,9 & 32,8 & 415 \\
\hline $\begin{array}{l}\text { Überzuckerung bei Dia- } \\
\text { betes }\end{array}$ & 2,7 & 10,8 & 46,3 & 40,2 & 415 \\
\hline Epileptischer Anfall & 4,4 & 16 & 32,4 & 47,2 & 413 \\
\hline Migräneattacke & 6,1 & 36,7 & 42,7 & 14,6 & 412 \\
\hline
\end{tabular}

\section{Sicherheit im Umgang mit} chronisch erkrankten

\section{Schüler/-innen}

Mehr als die Hälfte der Befragten zeigt bei 5 der 7 aufgelisteten Erkrankungen einen unsicheren Umgang (sehr unsicher oder eher unsicher; - Tab.4). Die größte Unsicherheit besteht bei einer Depression $(80,3 \%)$ und bei Diabetes mellitus (74,4\%). Die größte Sicherheit dagegen besteht bei Neurodermitis $(50,4 \%)$ und ADHS (55,3\%).

\section{Sicherheit in Notfallsituationen bei Schüler/-innen mit chronischen Erkrankungen}

Bei allen genannten Notfallsituationen geben die Lehrkräfte Unsicherheiten an (•Tab. 4). Die größte Unsicherheit besteht bei einer Notfallsituation vor dem Hintergrund eines angeborenen Herzfehlers (nur 7,3\% der Befragten fühlen sich sehr sicher oder eher sicher). Am sichersten fühlen sich Lehrkräfte im Umgang mit einer Migräneattacke bei
Schüler/-innen (42,8\% der Befragten fühlen sich sehr sicher oder eher sicher).

\section{Zusammenhang zwischen Gesundheitskompetenz und Sicherheit im Umgang mit Notfallsituationen bzw. mit chronisch erkrankten Schüler/-innen}

Eine multiple Regressionsanalyse zeigte einen statistisch signifikanten $\mathrm{Zu}$ sammenhang zwischen der Gesundheitskompetenz und der Sicherheit im Umgang mit chronisch erkrankten Schulkindern (- Tab. 5) und der Sicherheit im Umgang mit Notfallsituationen bei chronisch erkrankten Schulkindern (• Tab.6). Demnach führt jeder zusätzliche Punkt auf dem HLS-EU-Q16 zu einem Anstieg in der selbsteingeschätzten Sicherheit im Umgang mit chronisch kranken Kindern um 0,8 Punkte und im Umgang mit Notfällen um 0,4 Punkte (- Tab. 5 und $\bullet$ Tab. 6). Vor dem Hintergrund jedoch, dass es sich in der Studie um Selbsteinschätzungen von Kompe- tenzen der befragten Lehrkräfte handelt, sollte der Effekt eher zurückhaltend interpretiert werden. Alter und Geschlecht sind keine signifikanten Prädiktoren der Sicherheit im Umgang. Insgesamt erklären die Modelle nur einen kleinen Teil der Varianz $\left(\mathbf{R}^{2}\right)$.

\section{Diskussion}

Die vorliegende Studie ging der Frage nach, wie hoch die Gesundheitskompetenz von Lehrkräften an allgemeinbildenden Schulen in den Bundesländern Brandenburg und Hessen ist und ob im Umgang mit chronisch erkrankten Schüler/-innen bzw. in Notfallsituationen Unsicherheiten bei den Lehrkräften bestehen. Sowohl aus bildungspolitischer als auch aus gesundheitswissenschaftlicher Sicht sind die gewonnenen Erkenntnisse höchst relevant, da zum einen das Setting Schule in den Fokus von Gesundheitsförderung rückt und zum anderen Inklusion von gesundheitlich benachteiligten Kindern und Jugendlichen immer bedeutsamer wird. Zudem ist die Lehrergesundheit sowohl Determinante als auch Ergebnis einer gesunden Lebenswelt Schule.

Die Hälfte der befragten Lehrkräfte weist eine eingeschränkte Gesundheitskompetenz auf. Die meisten selbsteingeschätzten Schwierigkeiten treten bei Fragen zur Prävention auf. Besonders schwer fällt den Befragten der Umgang mit Themen der psychischen Gesundheit, was vor dem Hintergrund zunehmender psychischer Erkrankungen bei Kindern und Jugendlichen bedenkenswert erscheint. Darüber hinaus konnte eine Unsicherheit bei der Beurteilung der Vertrauenswürdigkeit digitaler Informationsquellen festgestellt werden. Hierbei ist jedoch festzuhalten, dass diese Einschätzung der Lehrkräfte eher auf einen kritischen Umgang ihrerseits mit solchen Informationen zurückzuführen ist als auf eine niedrige Gesundheitskompetenz. Weiterhin begegnen die Befragten den aufgelisteten Notfallsituationen sowie dem Umgang mit chronisch erkrankten Schüler/-innen mit überwiegender Unsicherheit. Eine niedrige Gesundheitskompetenz geht mit einer größeren Unsicherheit im 
Tab. 5 Zusammenhang zwischen der Gesundheitskompetenz und der Sicherheit im Umgang mit chronisch kranken Schulkindern $(n=355)$

\begin{tabular}{|c|c|c|c|}
\hline \multirow[t]{2}{*}{$\begin{array}{l}\text { Unabhängige } \\
\text { Variablen }^{\mathrm{a}}\end{array}$} & \multicolumn{3}{|c|}{$\begin{array}{l}\text { Abhängige Variable: Sicherheit im Umgang mit chronisch kranken } \\
\text { Schüler/-innen }\end{array}$} \\
\hline & Model 1 & Model 2 & Model 3 \\
\hline Konstante & $16.685^{* * *}$ & $16.214^{* * *}$ & $19.165^{* * *}$ \\
\hline $\begin{array}{l}\text { Gesundheits- } \\
\text { kompetenz }\end{array}$ & $0,822^{* * *}$ & $0,819^{* * *}$ & $0,814^{* * *}$ \\
\hline Alter & 0,061 & 0,063 & - \\
\hline Geschlecht & 0,336 & - & - \\
\hline \multicolumn{4}{|l|}{ Güte des Modells } \\
\hline Adjusted $\mathrm{R}^{2}$ & 0,080 & 0,082 & 0,077 \\
\hline
\end{tabular}

Tab. 6 Zusammenhang zwischen der Gesundheitskompetenz und der Sicherheit im Umgang mit Notfallsituationen bei chronisch kranken Schulkindern $(n=364)$

\begin{tabular}{|c|c|c|c|}
\hline \multirow[t]{2}{*}{$\begin{array}{l}\text { Unabhängige } \\
\text { Variablen }^{\mathrm{a}}\end{array}$} & \multicolumn{3}{|c|}{$\begin{array}{l}\text { Abhängige Variable: Sicherheit im Umgang mit Notfallsituationen bei } \\
\text { chronisch kranken Schüler/-innen }\end{array}$} \\
\hline & Model 1 & Model 2 & Model 3 \\
\hline Konstante & $7,121^{* * *}$ & $8,645^{* * *}$ & $9,646^{* * *}$ \\
\hline $\begin{array}{l}\text { Gesundheits- } \\
\text { kompetenz }\end{array}$ & $0,435^{* * *}$ & $0,442^{* * *}$ & $0,440^{* * *}$ \\
\hline Alter & 0,027 & 0,021 & - \\
\hline Geschlecht & 1,072 & - & - \\
\hline \multicolumn{4}{|l|}{ Güte des Modells } \\
\hline Adjusted $\mathrm{R}^{2}$ & 0,063 & 0,057 & 0,058 \\
\hline
\end{tabular}

Umgang mit Gesundheitsproblemen der Schüler/-innen einher.

Die Ergebnisse zur Gesundheitskompetenz in dieser Studie zeigen Ähnlichkeiten mit bisherigen Untersuchungen aus Deutschland auf (z.B. HLS-EU, GEDA, WIdO, HLS-GER; [9, 36-38]). Aufgrund der Verwendung desselben Messinstruments (HLS-Q16) sowie der Unterteilung in Subgruppen (u.a. Bildungsniveau) werden als Referenzwerte die Ergebnisse der Studie „Gesundheit in Deutschland aktuell“ (GEDA 2014; [37]) genutzt. Vergleicht man die Werte der Lehrkräfte mit den Werten der Subgruppe "hohes Bildungsniveau“ (darin u.a. Akademiker) aus der GEDA-Studie, so zeigen die Lehrkräfte schlechtere Werte als die Referenzpopulation (50,1\% der Lehrkräfte weisen ein ausreichendes GK-Level auf, bei der Referenzpopulation sind es 59,6\%; - Tab. 2). Im Vergleich $\mathrm{zu}$ den Lehrkräften fällt es der Referenzpopulation besonders schwer,
Informationen $\mathrm{zu}$ „beurteilen“ und $\mathrm{zu}$ „finden“, gefolgt von den Dimensionen „Verstehen“ und „Anwenden“.

Die Lehrkräfte gaben in der vorliegenden Studie an, besonders große Schwierigkeiten $\mathrm{zu}$ haben, Informationen zur Verbesserung ihres psychischen Wohlbefindens zu finden. Das kann sich nachteilig auf ihre eigene mentale Gesundheit auswirken, etwa wenn psychische Gefährdungen nicht rechtzeitig erkannt und angegangen werden. Gleichzeitig fällt es den Lehrkräften schwerer, psychische Probleme bei Schüler/-innen und deren Familien rechtzeitig zu erkennen und entsprechende Schritte der Unterstützung, z. B. durch Schulgesundheitspflegende, einleiten zu können. In der Vergangenheit wurde bereits gezeigt: Je höher die soziale Unterstützung durch Lehrkräfte, desto höher ist die Lebenszufriedenheit und desto geringer das Ausmaß an psychosomatischen Beschwerden bei Schüler/-innen [18, 39].
Bei mangelnder sozialer Unterstützung hingegen werden depressive Symptome und Ängste bei Schüler/-innen beobachtet. Auffällig ist in unserer Studie der vergleichsweise hohe Anteil fehlender Werte $(10 \%)$ bei den Items zu psychischen Unterstützungsmöglichkeiten (- Tab. 3, Q8). Hier liegen möglicherweise mangelnde Erfahrungen der Befragten zugrunde, die zu Unsicherheiten und Wissensdefiziten führen können. Zudem betrifft diese Frage womöglich eine sensitive persönliche Thematik, die zur Nichtbeantwortung dieser Frage geführt hat $[40,41]$.

Die Ergebnisse lassen vermuten: Unsicherheiten aufseiten der Lehrkräfte, die z.B. Fragen der Prävention, der eigenen psychischen Gesundheit oder der Vertrauenswürdigkeit digitaler Informationsquellen betreffen, beeinflussen ihre individuelle Gesundheitskompetenz. Hier gilt es zukünftig, Defizite in diesen Bereichen zu verringern. Denn je gesundheitskompetenter Lehrkräfte selbst sind, desto sicherer fühlen sie sich auch im Umgang mit gesundheitlich benachteiligten Schüler/-innen. Die selbst wahrgenommene Sicherheit ist auf die individuellen Fähigkeiten der Lehrkräfte, insbesondere in der Dimension „Anwenden“ zurückzuführen. Nach den Definitionen von Rudd und Anderson [6] sowie Kickbusch und Maag [7] sind jedoch nicht ausschließlich individuelle Kompetenzen bzw. Fähigkeiten für das Niveau von Gesundheitskompetenz ausschlaggebend, sondern auch situative Anforderungen bzw. die soziale Umwelt. Diese Kontextabhängigkeit zeigt sich im vorliegenden Fall im Setting Schule als Arbeitsplatz der Lehrkräfte und Ort der sozialen Interaktion mit den (teils auch gesundheitlich beeinträchtigten) Schüler/-innen. Die hohen situativen Anforderungen im Berufsalltag der Lehrkräfte nehmen somit Einfluss auf das Ergebnis ihrer individuellen Gesundheitskompetenz. Schlussfolgernd sind womöglich $\mathrm{zu}$ hohe situative Anforderungen im Setting Schule sowie teilweise eingeschränkte individuelle Fähigkeiten bzw. Kompetenzen verantwortlich für eine unzureichende Gesundheitskompetenz und somit für die 
großen Unsicherheiten im Umgang mit chronisch erkrankten Schüler/-innen.

Unter Einbezug des konzeptuellen Modells nach Sørensen et al. [8] lässt sich das Phänomen folgendermaßen erläutern: Lehrkräfte wenden ihr individuelles Wissen und ihre Kompetenzen an, um, im Idealfall, einen sicheren Umgang mit chronisch erkrankten Schüler/-innen zu gewährleisten. Dabei finden situative, persönliche sowie gesellschaftlich- und umweltbedingte Determinanten auf der individuellen Ebene Anwendung, um Herausforderungen auf der Bevölkerungsebene, nämlich den Schüler/-innen, zu begegnen. Durch ihre Vorbildfunktion und ihre Unterstützungsmöglichkeiten könnten Lehrkräfte Einfluss auf das Gesundheitsverhalten ihrer Schüler/-innen nehmen, welches sich langfristig auswirken kann, etwa auf den Gesundheitszustand im Erwachsenenalter und damit verbunden auf die Inanspruchnahme von Leistungen des Gesundheitssystems. Außerdem könnte die Gesundheitskompetenz von Lehrkräften gesundheitliche Chancengleichheit, Partizipation sowie Empowerment von gesundheitlich benachteiligten und nichtbenachteiligten Kindern und Jugendlichen fördern. Mit Blick auf Sørensen et al. kann auch hier von einer Wechselwirkung zwischen individuellen Determinanten und den Determinanten auf der Bevölkerungsebene ausgegangen werden.

Neben Themen des psychischen Wohlbefindens stellen auch Medien als Informationsquelle häufig große Schwierigkeiten für Lehrkräfte dar (• Tab. 3, Q8, Q11, Q12, Q13). Das unterstreicht, dass nicht nur der Medienkompetenz der Heranwachsenden, sondern auch der der Lehrkräfte mehr Aufmerksamkeit gewidmet werden sollte.

Stärken der vorliegenden Studie sind eine vergleichsweise große Stichprobe aus einem gesundheitswissenschaftlich relevanten Setting. Die beteiligten Schulen bilden unterschiedliche Schultypen aus dem deutschen Bildungssystem ab, darunter Grund- und Sekundarschulen, sowohl aus dem ländlichen als auch dem städtischen Raum.

Limitiert werden die Ergebnisse dieser Studie durch das Querschnittsdesign, welches zwar signifikante Korrelationen, aber keine Kausalitäten aufzeigen kann.

\section{Fazit}

Die Gesundheitskompetenz von Lehrkräften sollte gestärkt werden, um einerseits deren eigene Gesundheit zu erhalten und zu verbessern, insbesondere im Umgang mit berufstypischen Belastungen, aber auch um die Gesundheitskompetenz und damit die Gesundheitschancen der Schüler/-innen zu steigern. Insbesondere Ansätze zur Förderung der mentalen Gesundheit, aber auch die schwierige Aufgabe der Inklusion von Kindern und Jugendlichen mit chronischen Erkrankungen in den Schulalltag stellen zentrale Themen der schulischen Gesundheit dar.

In diesem Zusammenhang wären eine konzeptuelle Entwicklung sowie Erprobung von Maßnahmen zur gezielten Förderung des psychischen Wohlbefindens von Lehrkräften wünschenswert. Zudem könnte bedarfs- sowie bedürfnisorientiert auf die speziellen Anforderungen, auch im Hinblick auf die Inklusionsbestrebungen und den epidemiologischen Wandel bei Kindern und Jugendlichen eingegangen werden.

\section{Korrespondenzadresse}

\section{PD Dr. Antje Tannen, MPH}

Institut für Gesundheits- und Pflegewis-

senschaft, Charité Universitätsmedizin

Berlin

Berlin, Deutschland

antje.tannen@charite.de

Funding. Open Access funding provided by Projekt DEAL.

\section{Einhaltung ethischer Richtlinien}

Interessenkonflikt. A. Hartmann, J. Rückmann und A. Tannen geben an, dass kein Interessenkonflikt besteht.

Für die Datenerhebung und Auswertung im Rahmen der wissenschaftlichen Begleitforschung lag ein positives Votum der Ethikkommission der Deutschen Gesellschaft für Pflegewissenschaft vor (Antrag Nr. 16025) sowie die Zustimmung der in den jeweiligen Bundesländern zuständigen Ministerien.

Open Access Dieser Artikel wird unter der Creative Commons Namensnennung 4.0 International Lizenz veröffentlicht, welche die Nutzung, Vervielfältigung, Bearbeitung, Verbreitung und Wiedergabe in jegli- chem Medium und Format erlaubt, sofern Sie den/die ursprünglichen Autor(en) und die Quelle ordnungsgemäß nennen, einen Link zur Creative Commons Lizenz beifügen und angeben, ob Änderungen vorgenommen wurden.

Die in diesem Artikel enthaltenen Bilder und sonstiges Drittmaterial unterliegen ebenfalls der genannten Creative Commons Lizenz, sofern sich aus der Abbildungslegende nichts anderes ergibt. Sofern das betreffende Material nicht unter der genannten Creative Commons Lizenz steht und die betreffende Handlung nicht nach gesetzlichen Vorschriften erlaubt ist, ist für die oben aufgeführten Weiterverwendungen des Materials die Einwilligung des jeweiligen Rechteinhabers einzuholen.

Weitere Details zur Lizenz entnehmen Sie bitte der Lizenzinformation auf http://creativecommons.org/ licenses/by/4.0/deed.de.

\section{Literatur}

1. Schaeffer D, Dierks M-L (2016) Patientenberatung. In: Hurrelmann K, Razum $\mathrm{O}$ (Hrsg) Handbuch Gesundheitswissenschaften, 6. Aufl. Beltz Juventa Weinheim/Basel, S757-790

2. Schaeffer D, Pelikan J (2017) Health literacy: Begriff, Konzept, Relevanz. In: Schaeffer D, Pelikan J (Hrsg) Health Literacy: Forschungsstand und Perspektiven. Hogrefe, Bern, S11-18

3. Nutbeam D (1998) Evaluating health promotionprogress, problems and solutions. Health Promot Int 13:27-44

4. Nutbeam D (2000) Health literacy as a public health goal: a challenge for contemporary health education and communication strategies into the 21st century. Health Promot Int 15:259-267

5. Nutbeam D (2008) The evolving concept of health literacy. Soc Sci Med 67:2072-2078

6. Rudd RE, Anderson JE (2006) The health literacy environment of hospitals and health centers. Partners for action: making your healthcare facility literacy-friendly. National Center for the Study of Adult Learning and Literacy (NCSALL), Boston

7. Kickbusch I, Maag D (2008) Health literacy. In: Heggenhougen K, Quah S (Hrsg) International encyclopedia of public health. Elsevier, San Diego

8. Sørensen K, Van den Broucke S, Fullam J et al (2012) Health literacy and public health: a systematic review and integration of definitions and models. BMC Public Health 12(1):80

9. Schaeffer D, Vogt D, Berens E-M, Hurrelmann K (2016) Gesundheitskompetenz der Bevölkerung in Deutschland - Ergebnisbericht. Universität Bielefeld, Bielefeld

10. Schaeffer D, Hurrelmann K, Bauer U, Kolpatzik K (2018) Nationaler Aktionsplan Gesundheitskompetenz. Die Gesundheitskompetenz stärken. KomPart, Berlin

11. Hurrelmann K, Bauer U, Schaeffer D (2018) Strategiepapier \#1 zu den Empfehlungen des Nationalen Aktionsplans. Das Erziehungs- und Bildungssystem in die Lage versetzen, die Förderung von Gesundheitskompetenz so früh wie möglich im Lebenslauf zu beginnen. Nationaler Aktionsplan Gesundheitskompetenz 2018, Berlin

12. Okan O, Pinheiro P, Zamora P, Bauer U (2015) Health Literacy bei Kindern und Jugendlichen. Ein Überblick über den aktuellen Forschungsstand. Bundesgesundheitsblatt Gesundheitsforschung Gesundheitsschutz 58:930-941 
13. Zamora P, Pinheiro P, Okan O et al (2015) ${ }_{\text {"Health Literacy }}$ im Kindes- und Jugendalter. Präv Gesundheitsf 10:167-172

14. Lamanauskas V (2018) Teacher health literacy: Why does it matter? Problems of Education in the 21st Century. https:// www.researchgate.net/profil/Vincentas Lamanauskas/publication/323525109_Teacher_ health_literacy_Why_does_it_matter/links/ 5a998d3fa6fdcc3cbac924f2/Teacher-healthliteracy-Why-does-it-matter.pdf. Zugegriffen: 27. Aug. 2019

15. Paakkari L, Paakkari O, Villberg J, Tynjälä J, Ojala K, Välimaa R (2017) The association between teacher academic support and health literacy among 13- and 15-year olds: Finnish HBSC-study. http:// www.eera-ecer.de/ecer-programmes/pdf/print/ conference/22/contributi- on/41057/.. Zugegriffen: 10. Dez. 2017

16. Velardo S, Drummond M (2015) Teacher health literacy: the importance of multiple healthy role models within the school environment. 29th ACHPER international conference. Prince Alfred College, Adelaide

17. Stein M, Carey W, Friedman S et al (1993) The pediatrician and the "new morbidity" — committee on psychosocial aspects of child and family health. Pediatrics 92(5):731-733

18. Bilz L, Sudeck G, Bucksch J et al (2016) Schule und Gesundheit. Ergebnisse des WHO-Jugendgesundheitssurveys "Health Behaviour in School-aged Children". Gesundheitsforschung. Beltz JuventaVerlag, Weinheim, Basel

19. Hummrich M, Kramer R-T (2017) Schulische Sozialisation. Basiswissen Sozialisation. Springer VS, Wiesbaden

20. HBSC-Studienverbund Deutschland (2015) Studie Health Behaviour in School-aged Children. http:// www.gbe-bund.de.Zugegriffen:27.Aug. 2019

21. Klasen F, Meyrose AK, Otto C, Reiß F, RavensSieberer U (2017) Psychische Auffälligkeiten von Kindern und Jugendlichen in Deutschland. Ergebnisse der BELLA-Studie. Kinderheilkunde 165(5):402

22. Poethko-Müller C, Kuntz B, Lambert T, Neuhauser H (2018) Die allgemeine Gesundheit von Kindern und Jugendlichen in Deutschland - Querschnittsergebnisse aus KiGGS Welle 2 und Trends. J Health Monit. https://doi.org/10.17886/RKI-GBE-2018007

23. Bruland D, Kornblum K, Harsch S, Bröder J, Okan O, Bauer U (2017) Schüler mit einem psychisch erkrankten Elternteil und die Mental Health Literacy von Lehrkräften. Prax Kinderpsychol Kinderpsychiatr 66(10):774-790

24. Beauftragte der Bundesregierung für die Belange von Menschen mit Behinderungen (2017) UNBehindertenrechtskonvention. Übereinkommen über die rechte von Menschen mit Behinderungen. - Die amtliche, gemeinsame Übersetzung von Deutschland, Österreich, Schweiz und Lichtenstein. Beauftragte der Bundesregierung für die Belange von Menschen mit Behinderungen, Berlin

25. Kocks A (2008) Die Rolle der schwedischen School Health Nurse und das Thema Gesundheit im Setting Schule. Pflege Ges 13(3):246-260

26. Tannen A, Ebert J, Adam Y, Ewers M (2017) Gesunden Lebensstil früh fördern. Heilberufe 69:59-61

27. Paulus $P$ (2002) Gesundheitsförderung im Setting Schule. Bundesgesundheitsblatt Gesundheitsforschung Gesundheitsschutz 45:970-975

28. Scheuch K, Haufe E, Seibt R (2015) Lehrergesundheit. Dtsch Arztebl 112(20):347-356
29. Schumacher L, Nieskens B, Sieland B et al (2012) Handbuch Lehrergesundheit. Link, Köln

30. Dillmann M, Bensch S, Kocks A (2015) Das könnte Schule machen. JuKiP Fachmag Gesundh Kinderkrankenpfl 4(03):118-122

31. Kocks A (2008) Schulgesundheitspflege. Pflege Ges 3:246-260

32. Tannen A, Adam Y, Ebert J, Ewers M (2018) Schulgesundheitspflege an allgemeinbildenden Schulen Teil 1 - Analyse der Ausgangslage. Working Paper No. 18-03 der Unit Gesundheitswissenschaften und ihre Didaktik. Charité Universitätsmedizin Berlin, Berlin

33. Tannen A, Adam Y, Ebert J, Ewers M (2018) Schulgesundheitspflege an allgemeinbildenden Schulen: Teil 2 - Implementationsbegleitung und Wirkungsanalyse. Working Paper No. 18-03 der Unit Gesundheitswissenschaften und ihre Didaktik. Charité Universitätsmedizin Berlin, Berlin

34. Pelikan JM, Röthlin F, Ganahl K (2014) Measuring comprehensive health literacy in general populations: validation of instrument, indices and scales of the HLS-EU study. 6th Annual Health Literacy Research Conference. Ludwig Boltzmann Institute Health Promotion Research, Vienna

35. Röthlin F, Pelikan JM, Ganahl K (2013) Die Gesundheitskompetenz der 15-jährigen Jugendlichen in Österreich. Abschlussbericht der österreichischen Gesundheitskompetenz Jugendstudie im Auftrag des Hauptverbands der österreichischen Sozialversicherungsträger (HVSV)

36. Sørensen K, Pelikan JM, Röthlin F et al (2015) Health literacy in Europe: comparative results of the European health literacy survey (HLS-EU). Eur J Public Health 25(6):1053-1058

37. Jordan S, Hoebel J (2015) Gesundheitskompetenz von Erwachsenen in Deutschland. Bundesgesundheitsblatt Gesundheitsforschung Gesundheitsschutz 58(9):942-950

38. Zok K (2014) Unterschiede bei der Gesundheitskompetenz. Ergebnisse einer bundesweiten Repräsentativ-Umfrage unter gesetzlich Versicherten. WldO Monit 11:1-12

39. Paulus P (2010) Bildungsförderung durch Gesundheit. Bestandsaufnahme und Perspektiven für eine gute gesunde Schule. Juventa, Weinheim

40. Klusmann U (2011) Belastung und Beanspruchung im Lehrerberuf. Zwischen beruflicher Praxis und interdisziplinären Forschungsansätzen. In: Terhart E, Bennewitz H, Rothland M (Hrsg) Handbuch der Forschung zum Lehrerberuf. Waxmann, Münster, S814-820

41. Klusmann U, Richter D, Lüdtke O (2016) Teachers' emotional exhaustion is negatively related to students' achievement: Evidence from a large-scale assessment study. J Educ Psychol 108:1193-1203 